\title{
Web 3D Visualization of Noise Mapping for Extended INSPIRE Buildings Model
}

\author{
Lukáš Herman and Tomáš Řezník \\ Masaryk University, Faculty of Science, Department of Geography, \\ Laboratory on Geoinformatics and Cartography (LGC), \\ Kotlářská 2, 61137 Brno, Czech Republic \\ herman.lu@mail.muni.cz, tom@mail.muni.cz
}

\begin{abstract}
This paper deals with cross-domain environmental aspects of noise mapping which is commonly understood as a stand-alone initiative. Scientific core of this research lies in the integration of noise mapping with interoperable data sources, where the European Union's INSPIRE (INfrastructure for SPatial InfoRmation in Europe) Directive is nowadays one of the main.

Principles of noise mapping according to the END (European Noise Directive), OGC (Open Geospatial Consortium) CityGML specification, X3D (Extensible 3D) and application schemas in INSPIRE are depicted at the beginning. Special attention is then paid to the specifics of INSPIRE spatial data theme called buildings, including its extension possibilities. Motivations for 3D application schema of INSPIRE buildings are discussed while only 2D application schema is the legally binding one. On the other hand, 3D application schema is recommended by the INSPIRE Technical Guidelines. We also deal with OGC CityGML specification since it is the basis for the $3 \mathrm{D}$ application schema. We took a step forward to publish the results of noise mapping based on INSPIRE application schema through X3D technology on the Web. This enables to disseminate information on noise mapping to wide audience through next generation Web applications.

The paper proposes formalised methodology on X3D-based application development through the XSLT (eXtensible Stylesheet Language Transformations). Noise mapping was taken as a use case. There are discussions on benefits and limitations of X3D visualization as well as differences between the $\mathrm{X} 3 \mathrm{D}$ presentation and storage of 3D geospatial data according to INSPIRE application schema on buildings at the end.
\end{abstract}

Keywords: geographic information, CityGML, X3D, INSPIRE, noise mapping.

\section{Introduction}

Geographic information has always been crucial for research in a wide variety of sciences within and beyond Geography. On the other hand, contemporary Web search engines are only partly suitable for efficient discovery and presentation of geographic information. The main difference between geographic and other data is the spatiotemporal dimension of geographic data. For that reason it is not possible to search for 
geographic data for a certain area using search engines such as Google, Yahoo, Bing, etc. Furthermore, such a limitation of geographic information discovery limits the reuse of geographic information [11].

To deal with this problem, the European Commission started the initiative called INSPIRE (INfrastructure for SPatial InfoRmation in Europe). This initiative was transformed in 2007 into the Directive of the European Commission and the Council with designation 2007/2/EC. This Directive was transposed into national legal systems of the EU (European Union) Member states between 2007 and 2009. The Directive itself contains the general concept, while more detailed information may be found in the corresponding Commission Regulations addressing specific issues, as well as in the underlying technical guidelines.

These are, so far, interoperability of spatial data, metadata of spatial data and services, data and service sharing, network services. The directive defines 34 spatial data themes covering a wide range from coordinate reference systems, cadastral parcels, transport networks, hydrography, land cover, orthoimagery, soil, human health and safety, natural risk zones, habitats and biotopes, energy resources, buildings, and many others.

We may also define interoperability on the conceptual and policy levels. INSPIRE Directive is closely related to other European Directives, international standards and standardization activities, etc. We may find examples in the linkages to European Noise Directive, Water Framework Directive, International Organisation for Standardization (ISO) 19100 series of standards for geographic information or implementation specifications of the Open Geospatial Consortium (OGC). This paper analyses the issues of the INSPIRE application schema for spatial data theme Buildings, its connection to the European Noise Directive, presentation and storage of 3dimensional geographic information created through innovative Web technologies with noise mapping as a use case.

\section{$2 \quad$ Legal and Standardization Framework}

\subsection{INSPIRE Application Schemas for Buildings}

The INSPIRE concept of geographic data follows an object-oriented approach of modelling the entities of the real world. This means that one or more application schemas covering each specific point of view(s) on the domain are created. Each application schema then contains explicit definitions of feature types, their aggregation into classes, attributes of feature types, domains of these attributes, etc. The primary aim is to develop a model which will ensure interoperability and harmonisation within each spatial data topic. Each application schema is offered in a number of ways, such as through a UML (Unified Modelling Language) class diagram, feature catalogue, XSD (eXtensible Stylesheet Document), and, of course, textual descriptions. Besides the application schemas themselves, the concept of INSPIRE geographic data also includes related aspects. These are definitions of the reference systems (coordinate, vertical as well as temporal), quality of geographic data, metadata and many others. On the other hand, common issues for all spatial data topics are written in the Generic Conceptual Model (GCM) document, which comprises definitions of unique 
identifiers for geographic data, explicit definition of data types, principles of network application schemas, multi-lingual geographic information, etc.

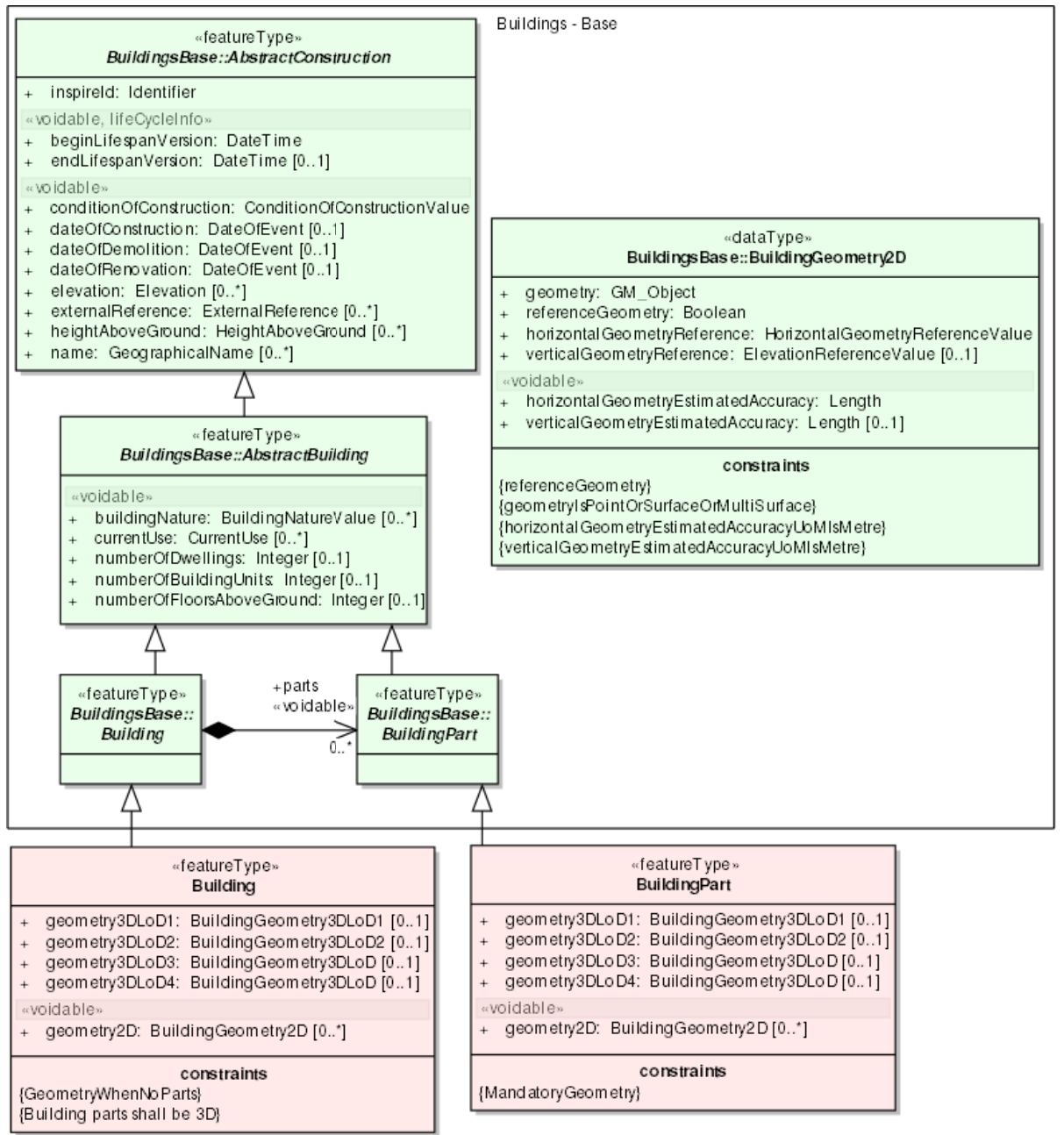

Fig. 1. UML class diagram for the INSPIRE application schema Buildings $3 D$ according to the INSPIRE Data Specification on Buildings - Draft Technical Guidelines [8].

The INSPIRE application schema for buildings is ${ }^{1}$ in its almost final phase, designated as release candidate 3 version. There may be minor changes in this version if the Amendment to the Commission Regulation No 1089/2010 will be modified. The latest version of the INSPIRE application schema for buildings mainly focuses on the physical description of real world entities seen as constructions. A capability of a building to provide services is a subject of other INSPIRE application schemas in 
another spatial data themes, above all utility and governmental services, production and industrial facilities, agricultural and aquacultural facilities. We may define four application schemas within the Buildings spatial data theme:

- The Buildings2D profile includes various geometrical representations of buildings as $2 \mathrm{D}$ or $2,5 \mathrm{D}$ data.

- The Buildings3D profile has same semantic content as the Buildings2D profile and allows in addition, the geometric representation of buildings in any of the four levels of detail of CityGML.

- The BuildingsExtended2D profile is a semantic extension of Buildings2D profile with additional thematic attributes (material of construction, official area or value, connection to utility networks ...), classes (building units, installations, other constructions) and references to other data (like cadastral data and addresses).

- The BuildingsExtended3D profile is an extension of the Buildings3D profile for rich $3 \mathrm{D}$ representations at different levels of details. It includes the possibility to represent many building components, such as the building boundaries (wall, roof), the openings (doors - windows) and building interior (rooms, internal installations) and the textures associated with the main feature types. It also contains all the semantic information of extended 2D profile.

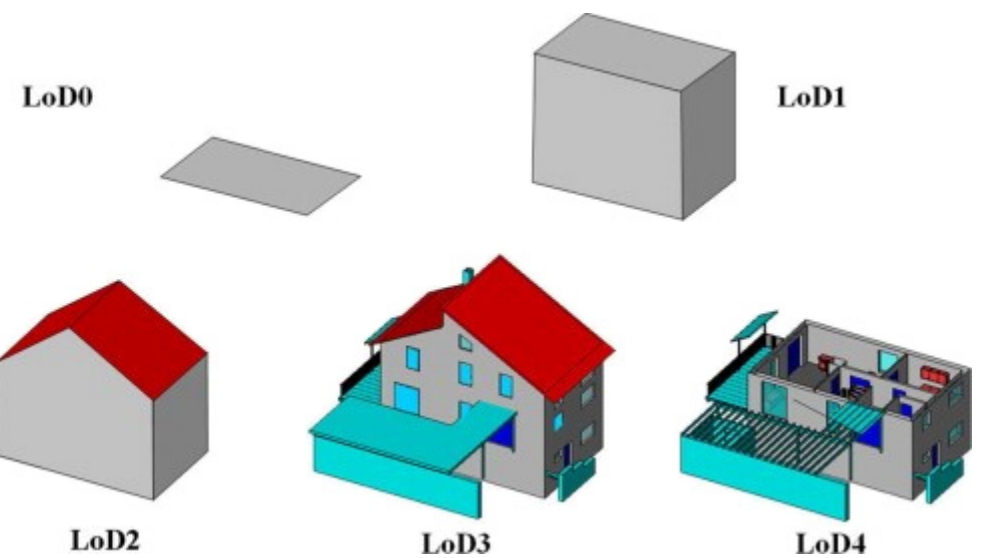

Fig. 2. Levels of detail (LoD) within the OGC CityGML implementation specification according to Data for 3D project, 2013 [3]. Please note, that INSIPRE 3D buildings application schemas incorporates only LoD1 to LoD4, since LoD0 is reserved for the 2D application schemas.

We will hereinafter deal only with the Buildings3D profiles since they are based on the OGC CityGML specification, enable to link to noise data and allow presenting through innovative X3D Web technology. Figure 1 contains the UML class diagram of the 3D application schema for buildings as a common starting point. Data type BuildingGeometry $2 D$ on the right side of the Figure is originating from the $2 \mathrm{D}$ application schemas for buildings. The pink bottom part enables to incorporate any building or even any part of the building in four levels of details (LoD) as depicted in 
Figure 2. This application schema also enables to aggregate information from buildings and building parts into two feature types: AbstractBuilding and AbstractConstruction.

\subsection{European Noise Directive}

Omnipresent noise enters more and more often and intensively into human subconsciousness as a part of everyday life and only few realize how harmful it is. Long term influence of noise can cause not only hearing impairment, but also many other illnesses. In particular, it has negative psychological effect in the form of stresses, neuroses, etc. Negative effects of noise caused that another Directive of the European Union (EU) was developed.

Directive 2002/49/EC of the European Parliament and of the Council of 25 June 2002 relating to the assessment and management of environmental noise is commonly known as the Environmental Noise Directive and hereinafter is referred to as the 'END'.

The END requires Member States to undertake strategic noise mapping. It also refers to legislation regarding access to the information from strategic noise maps, since it requires "that the strategic noise maps ... are made available and disseminated to the public in accordance with relevant Community legislation ... and in conformity with Annex IV ... to this Directive".

Strategic noise map (and mapping) is formally defined by the END as ,,a map designed for the global assessment of noise exposure in a given area due to different noise sources or for overall predictions for such an area". For more information on the END please refer to the Directive itself [4] or scientific articles with this topic such as [14].

The Aarhus Convention has established the right of everyone to receive environmental information held by public authorities ("access to environmental information"). The parties to this important Convention, including all European Union (EU) Member States, are required to make the necessary provisions so that public authorities at national, regional or local level ensure that this right is upheld.

Together the END (2002/48/EC), INSPIRE Directive (2007/2/EC), Directive on public access to environmental information (PSI; 2003/4/EC) and Aarhus convention have established two distinct aspects with regard to informing the public; (1) the availability of noise information upon request, and (2) the active and systematic dissemination of noise information to the public. We may identify the following chain in noise mapping: the END defines the noise mapping itself, the INSPIRE Directive spatial representation and discovery mechanisms and the PSI Directive re-use of data and information that was created within the END and INSPIRE.

\section{Technological framework}

\subsection{CityGML}

CityGML (City Geography Markup Language) is an open data model and XMLbased format for sets of 3D urban objects. It is implemented as an application schema 
for the GML3 (Geography Markup Language version 3). Both are extendible international standards for spatial data exchange issued by the OGC (Open Geospatial Consortium). CityGML is used for the representation, storage, and exchange of virtual 3D city models. Generalization hierarchies between thematic classes, aggregations and relations between objects are also included in this application schema. CityGML standard has been used as well as a basis for developing INSPIRE application schemas for spatial data theme Buildings.

CityGML provides a standard model and mechanisms for describing 3D objects with respect to their geometry, topology, semantics and appearance; furthermore defines five discrete levels of detail (LoD) as depicted in Figure 2. CityGML provides much more than $3 \mathrm{D}$ content for visualization to diverse applications. CityGML provides class definitions, regulations and explanations of the semantics for the important geographic features in virtual 3D city models including digital terrain models, buildings, water bodies, vegetation, transportation objects and city furniture [15]. CityGML has been designed as a universal topographic information model according to [10].

Practical applications often require storing and exchanging data, which do not belong to any of the predefined classes. CityGML provides two different ways of extension for such cases. The first one is a usage of generic features and generic attributes; however this method reduces semantic interoperability. Concept called Application Domain Extension (ADE) is used to eliminate this problem. In contrast with generic objects and attributes, ADE has to be defined within a separate XML Schema definition. CityGML data model extended by an ADE has been used for example for noise mapping in North-Rhine Westphalia in Germany [2].

\subsection{X3D}

X3D (Extensible 3D) is an XML based 3D graphic format. X3D originates from another 3D graphical format - VRML (Virtual Reality Modelling Language). X3D provides all functionality included in the second version of VRML, which is commonly known as VRML97. XML encoding enables better integration of X3D and contemporary Web technologies. X3D supports 3D graphics and programmable shaders, 2D graphics, animations, spatialized audio and video, user interaction and navigation, networking, scripting and user-defined objects. Several profiles are defined for various levels of capabilities (e.g. X3D Core, X3D Interchange, X3D Immersive, which most closely matches VRML97, and X3D Full). X3D was approved as an ISO standard (ISO/IEC 19775) in 2004 [9].

JavaScript library for rendering the X3D scenes directly in a Web browser is called X3DOM. X3DOM is based on direct integration of X3D elements into the HTML (HyperText Markup Language) code. The main goal is to achieve X3D scene available for JavaScript DOM (Document Object Model), which allows modifying the 3D scene by only adding, removing or changing DOM elements. No specific plug-in is needed. Common JavaScript events, like onclick, on 3D objects are supported as well. There is also available an X3DOM runtime API, which provides a proxy object to programmatically read and modify runtime parameters. The API functions are available for resetting views, cycling cameras or changing navigation modes [1]. X3D 
elements can also be stored as part of external files; their connection is realized via the element Inline. Particular X3D elements can be clearly distinguished through their attribute $D E F$.

\section{$4 \quad$ Use Case}

Standardization frameworks, INSPIRE application schemas for Buildings and CityGML, are suitable for storing 3D data about buildings and noise pollution. On the other hand, X3D has a bigger potential for the visualization in Web environment. Therefore, it is necessary to deal with the ways of transformation from mentioned data standards into X3D a as first step. XSLT (Extensible Stylesheet Language Transformations) language has been used for these purposes.

\subsection{Input Data}

Technologies described in this paper have been tested on example of a Brno city district called Nový Lískovec, the Czech Republic. CityGML model consisting of building in LoD1, terrain model in LoD0 and noise maps stored as generic objects in LoD1 were used as input data. Geometry of buildings originally comes from stereophotogrammetry and terrain model as developed for the official Czech topographic database called ZABAGED. Noise maps show spatial distribution of equivalent continuous sound pressure level in the night (22:00 - 6:00) and in the daytime (6:00 22:00). These maps were calculated on basis of compendious traffic from land transport: road, railway and tram system transport. Model of noise emissions has been calculated according to the situation in year 2004 .

\subsection{XSLT}

XSLT is a language for transforming the XML documents into the differently structured XML documents and/or open data formats. The original document is not changed. However a new document is created - based on the content of an existing one. XSLT uses two types of input data: input datasets and templates (stylesheet). The XSLT processor takes one or more XML input documents together with one or more XSLT stylesheet modules, and processes them to produce an output document. The XSLT stylesheet contains a collection of template rules: instructions and other directives that guide the processor in the production of the output document. The main principle of transformation is in this case simplification of the semantic structure. Both, described and possible inputs (CityGML and INSPIRE application schemas for Buildings), are based on GML3, thus form of both stylesheets is very similar.

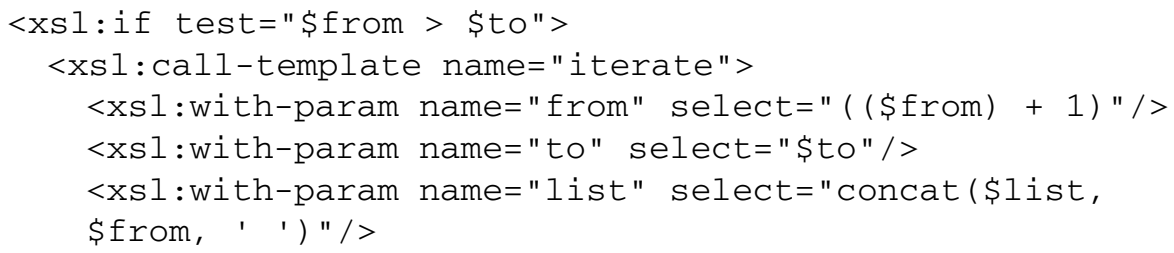




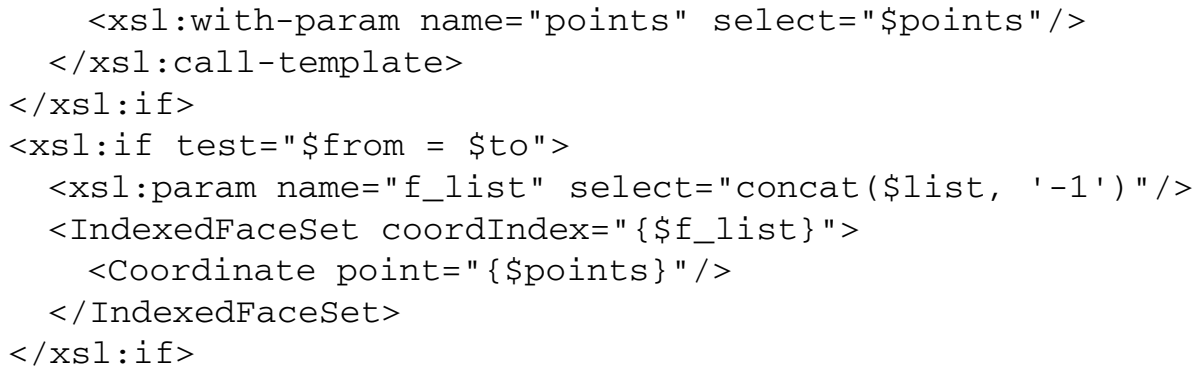

XSLT source code listing above depicts the list of coordinates $(\mathrm{X}, \mathrm{Y}, \mathrm{Z})$ which are transferred into the value of the attribute point. The attribute coordIndex contains sequence indicating the order in which the points are connected. These two key elements define geometry of element IndexedFaceSet, which defined the geometry of all volume and surface features.

\subsection{Differences between (City)GML and X3D}

The most significant difference between X3D and CityGML consists in the method of geometry storage. CityGML as well as all INSPIRE application schemas use a subset of the GML3 geometry models. Geometries of geographic features are in GML3 are represented as objects having an identity and further substructures. GML3 provides classes for geometric primitives (e.g. Point), composite geometries (CompositeSurface) and geometry aggregates (MultiSurface). Volumetric representations are modelled according to the Boundary representation (BRep), where each solid is bounded by a closed surface (typically CompositeSurface).

Geometry may be defined in X3D as a solid figure (elements Box, Cone, Cylinder, Sphere); while this method is not convenient for complex shapes of geographical features. Spatial vector data are represented in Geographical Information Systems (GIS) as points, lines and polygons. We may identify following X3D elements that are suitable for storing these types of spatial data: PointSet, IndexedLineSet and IndexedFaceSet. Raster data can be stored in X3D as ElevationGrid.

Definition of materials in CityGML is adopted from X3D, so there is not any issue with their transformation. Definitions of materials are in both cases composed of colour value, transparency and shines. For these purposes, X3D elements Material and ImageTexture are used. CityGML and X3D also allows linking to texture images. Since appearance is not inevitable part of input CityGML data, their definition may be a part of XSLT template. Appearance is not specified in the 3D profiles of INSPIRE application schemas for Buildings spatial data theme.

Semantic data structures are largely removed during the transformations, because X3D does not support storing attributes and semantic classes while enables only a general element (Group) for creating taxonomies. On the contrary, data originating from attributes can be stored in external files (e.g. XML or JSON) and connection would be provided through unique identificators (attributes $D E F$ or id) and selected programming language (e.g. JavaScript). The second type of semantic information connection is their storing in additional attributes. This method describes [16]. 


\subsection{Resulting Web Presentation}

Use case Web application consists of HTML page, JavaScript files and X3D files comprising digital terrain model, noise maps and buildings. Saving 3D data as X3D and using JavaScript and X3DOM library allow to implement interactivity such as interactive and non-interactive movement, setting up type of interactive movement, changing rendering mode and switching between noise maps for calculated for day and for night. Further enrichment of the user interface, which mentions [17], is also possible. Using internet technologies like X3DOM provides 3D visualization of spatial data, such as noise pollution, for wide audience.

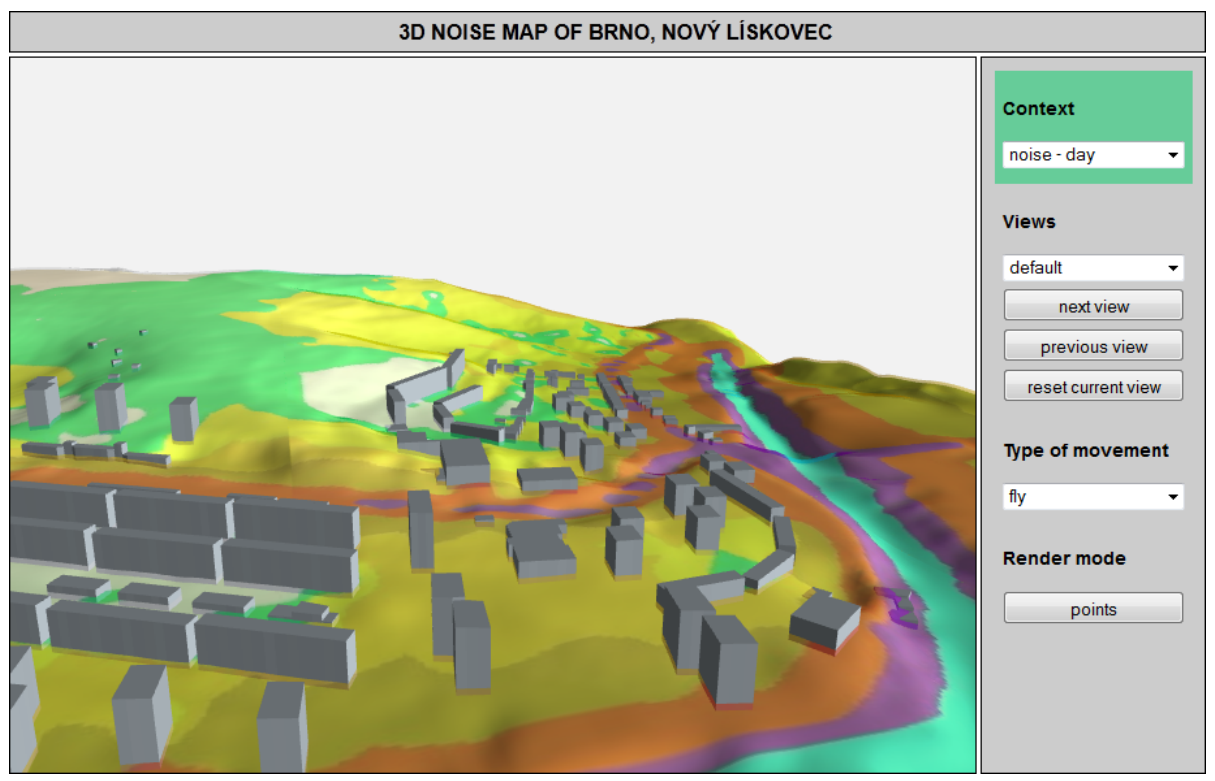

Fig. 2. Developed use case Web application for noise mapping with INSPIRE-based buildings. Visualization shows noise pollution in Brno city district Nový Lískovec, the Czech Republic

\section{$5 \quad$ Conclusions and Future Work}

It may be stated that the European legal frameworks of the END, INSPIRE and PSI directives represent complementary activities in the field of noise mapping. Models developed according to the END can be inserted into the models based on the INSPIRE application schemas and re-used in line with the principles of the PSI directive. The weakness of above mentioned legal frameworks lies in the lack of visualisation rules and best practices for the domain of noise mapping. A developed noise map shall follow strict rules for the data structure; however its visualization does not belong between the primary aspects. The same situation appears also in the documents of the INSPIRE directive: legally binding documents do not contain visualization principles. Visualization rules are formalised through the OGC Styled Layer Descrip- 
tor (SLD) in the INSPIRE technical guidelines; however any rule does not relate to a $3 \mathrm{D}$ profile.

We have therefore developed and tested X3D-based visualization in comparison to common Web services as depicted, for instance, by [7]. To sum up, we have identified four major advantages of the $\mathrm{X} 3 \mathrm{D}$ visualization for noise mapping:

- X3D enables user-friendly 3D visualization based on standardized Web technologies;

- X3D does not require to install any new software or plug-in;

- X3D enables to present noise maps calculated in 2D as 3D visualization;

- X3D enables exploration of barrier effect caused by high buildings blocks and/or other impacts of terrain and buildings.

Future work should aim at issues of noise mapping in 3D. Currently the END requires only the noise map for a level of 4 meters above the terrain, i.e. typically the second floor of a building. The X3D technology allows displaying a noise value at each part of a building. Such technique would be valuable for visualization of areas highly polluted by noise, such as city centres or built-up areas near highways. The first attempts on such noise mapping may be found in [12].

Acknowledgements. This research has been supported by funding from the project of Masaryk University under the grant agreement No. MUNI/A/0902/2012, which is called 'Expression of Global Environmental Change in Component Earth's Spheres'.

\section{References}

1. Behr, J., Jung, Y., Drevensek, T., Aderhold, A.: Dynamic and Interactive Aspects of X3DOM. In: Proceedings of the 16th International Conference on 3D Web Technology (2011)

2. Czerwinski, A., Sandmann, S., Stőcker-Meier, E., Plümer, L.: Sustainable SDI for EU noise mapping in NRW - best practice for INSPIRE. International Journal of Spatial Infrastructures Research 2, 90-111 (2007)

3. Data for 3D project, http://www.agency9.com/products/city-planner/ data-for-3d-project/

4. Directive 2002/49/EC of the European Parliament and of the Council of 25 June 2002 relating to the assessment and management of environmental noise - Declaration by the Commission in the Conciliation Committee on the Directive relating to the assessment and management of environmental noise,

http: / / eur-lex. europa . eu / LexUriServ/LexUriServ . do?uri=CELEX : 32002L0049: EN:HTML

5. Directive 2003/4/EC of the European Parliament and of the Council of 28 January 2003 on public access to environmental information and repealing Council Directive 90/313/EEC, http: / / eur-lex. europa.eu/LexUriServ/LexUriServ.do?uri=OJ : L:2003:041:0026:0032:EN:PDF (accessed April 25, 2013) 
6. Directive 2007/2/EC of the European Parliament and of the Council of 14 March 2007 establishing an Infrastructure for Spatial Information in the European Community (INSPIRE), http: / / eur-lex. europa . eu/LexUriServ/LexUriServ. do?uri=OJ : L : 2007:108:0001:0014:en:PDF

7. Horák, J., Ardielli, J., Růžička, J.: Performance Testing of Web Map Services. In: Nguyen, N.T., Trawiński, B., Jung, J.J. (eds.) New Challenges for Intelligent Information and Database Systems. SCI, vol. 351, pp. 257-266. Springer, Heidelberg (2011)

8. INSPIRE Data Specification on Buildings - Draft Technical Guidelines, http://inspire.jrc.ec.europa.eu/documents/Data_Specifications / INSPIRE_DataSpecification_BU_v3.0rc3.pdf

9. ISO/IEC 19776:2007 Information technology - Computer graphics and image processing - Extensible 3D (X3D) encodings, http: / /www . web3d.org/x3d/specifications / ISO-IEC-19776-X3DEncodings-All-Edition-1/

10. Kolbe, T.H.: Representing and exchanging 3D city models with CityGML. In: Lee, J., Zlatanova, S. (eds.) 3D Geo-information Sciences, pp. 15-31. Springer, Heidelberg (2009)

11. Konečný, M.: CARTOGRAPHY: Challenges and Potentials in Virtual Geographic Environments Era. Annals of GIS 17(3), 135-146 (2011)

12. Law, C., Lee, C., Lui, A.S., Yeung, M.K., Lam, K.: Advancement of three-dimensional noise mapping in Hong Kong. Applied Acoustics 72(8), 534-543 (2011)

13. Mao, B., Ban, Y.: Web-based Visualisation of the Generalised 3D City Models using HTML5 and X3DOM (2013),

http: / / jselab.njue.edu.cn/download/conference/

Web-based\%20Visualisation\%200f 20 the 20 Generalised\%203D\%

$20 \mathrm{Ci}$ ty 20 Models\%20using $20 \mathrm{HTML} 5 \% 20$ and\%20X3DOM.pdf

14. Murphy, E., King, E.A.: Strategic environmental noise mapping: Methodological issues concerning the implementation of the EU Environmental Noise Directive and their policy implications. Environment International 36(3), 290-298 (2010)

15. OGC City Geography Markup Language (CityGML) Encoding Standard, http: / / www . opengeospatial .org/standards / citygml

16. Pittarello, F., De Faveri, A.: Semantic Description of 3D Environments: a Proposal Based on Web Standards. In: Proceedings of the 11th International Conference on 3D Web Technology (2006)

17. Staněk, K., Friedmannová, L., Kubíček, P., Konečný, M.: Selected issues of cartographic communication optimization for emergency centers. International Journal of Digital Earth 3(4), 316-339 (2010) 